SUPPLEMENTARY MATERIALS

\title{
INTEGRATION OF CONDUCTIVITY, TRANSPARENCY, AND MECHANICAL STRENGTH INTO HIGHLY HOMOGENEOUS LBL COMPOSITES OF SWNT FOR OPTOELECTRONICS AND SOLAR ENERGY HARVESTING
}

\author{
Bong Sup Shim ${ }^{1}$, Zhiyong Tang ${ }^{1}$, Matthew P. Morabito', Ashish Agarawal ${ }^{1}$, Haiping Hong, \\ Nicholas A. Kotov ${ }^{1,2 *}$
}

SWNT Tightening by Thermal Annealing and Its Observation in LBL Films.

After the creation of percolation network, the improvement of the quality of SWNT-SWNT contacts is one of the most important requirements to the design of electrically conductive SWNT composites, because the efficiency of electron tunneling through the gap is exponentially dependent on the SWNT-SWNT distance. Therefore we tried molecular tightening by thermal annealing above polymer's glass transition temperature, even though high temperature treatments have negative impacts on the conductivity of polymeric LBL films because of reducing volatile admixture and ion doping effects and density increasing micro-Brownian motion. ${ }^{1}$ After a simple thermal annealing, the 200 layered sample showed significant improvements in its conductivity reaching $6.3 \pm 0.3 * 10^{3} \mathrm{~S} / \mathrm{m}$, which can be attributed to annealing of structural imperfections due to slightly increased molecular mobility. We further investigated the heat treatment effects by monitoring the electrical properties of $[\mathrm{PVA} /(\mathrm{SWNT}+\mathrm{PSS})]_{200}$ while gradually varying temperature in two cycles from $40^{\circ} \mathrm{C}$ to $200^{\circ} \mathrm{C}$ (Figure $3 \mathrm{~A}$ ). In addition to potential enhancement of charge transport, these

\footnotetext{
* corresponding author. E-mail: kotov@umich.edu.
} 
data will also provide information on thermal stability of the electrical properties. The electrical conductivity of $[\mathrm{PVA} /(\mathrm{SWNT}+\mathrm{PSS})]_{200}$ was initially $3 * 10^{3} \mathrm{~S} / \mathrm{m}$ at $40^{\circ} \mathrm{C}$ and increased to $4.15^{*} 10^{4} \mathrm{~S} / \mathrm{m}$ at $300^{\circ} \mathrm{C}$. (Figure 5) However, the electrical conductivity increase is reversed if the heat treatments at $200^{\circ} \mathrm{C}$ are prolonged or repeated (Figure S1). Here, we noticed that the sharp conductivity increase at $300^{\circ} \mathrm{C}$ might be associated with not only chemical/physical relaxation of the composite but also with irreversible structural rearrangement of SWNTs because the annealing process caused permanent changes in electrical properties. This is consistent with strengthening of van-der-Waals bonds with temperature, which is typically irreversible.

Considering the nature of the temperature effects on the composite structure, one can investigate the changes of van Hove singularity peaks in UV-vis spectroscopy before and after thermal treatments. Spectral positioning of van Hove singularities depends on SWNT diameters and specific chiral structures. ${ }^{2}$ So, if SWNTs are fully exfoliated as a single strand, absorption spectra of the SWNT mixture have discrete peaks corresponding to electronic excitation energy, e.g., $E_{11(\mathrm{~S})}, \mathrm{E}_{22(\mathrm{~S})}, \mathrm{E}_{11(\mathrm{M})}$ etc. Also, both $\mathrm{E}_{11(\mathrm{~S})}$ and $\mathrm{E}_{22(\mathrm{~S})}$ peaks of semiconducting SWNTs can provide useful information about inner organization of the composites. As such, $\mathrm{E}_{22(\mathrm{~S})}$ peaks or peaks in the visible light range are less sensitive to chemical doping changes around SWNTs than $\mathrm{E}_{11(\mathrm{~S})}$ peaks ${ }^{3,4}$. Therefore, only $\mathrm{E}_{22(\mathrm{~S})}$ or higher energy peaks were examined for the transparent 8,12 , and 16 layered films of [PVA/(SWNT+PSS)]. Existence of these van Hove singularity peaks is a distinct criterion of 
the "goodness" of the SWNT solvent as well as the quality of the SWNT dispersions. ${ }^{5}$ If these SWNTs are bundled, then down shifting and broadening of the peaks can be expected due to band gap lowering inter-tube interactions. ${ }^{6}$ In the solid composite system these peak shifting and broadening effects become a prominent evidence of molecularly controlled SWNT rearrangements (Figure S2) leading to tighter connection between SWNTs. Also, thermal annealing does not produce any change in macro-appearances at all, but these are only monitored by the shape and shift of the van Hove transition peaks.

\section{SWNT Concentration Effect during LBLAssemblies and Its Electrical Properties.}

The solution quality of LBL assemblies affects the layered structure. For example, if the concentration of a SWNTs dispersion solution is diluted, then the adsorbed amount of SWNT in a given amount of time dipping decreases and the material properties of the resulting composite changes. Here, we compared the optical properties of LBL assemblies with varied SWNT concentration solutions and the electrical properties of the resulting 10 layered films before and after heat treatment at $300^{\circ} \mathrm{C}$ for 10 mins. (Figure $\mathrm{S} 3$ )

\section{X-ray Diffraction Study of 200 layered SWNT LBL films}

Wide-angle and small-angle X-ray diffraction (WXRD, SXRD) were performed to 200 layered SWNT LBL films. Unlike aligned SWNT fibers, the peak of both WXRD and SXRD scans was not very obvious because the arrangements of SWNTs are layered and 2 dimensionally isotropic. The slight broad hills in WXRD corresponded to $1.47 \mathrm{~nm}$ and 0.44 
$\mathrm{nm}$ respectively. Absence of peaks at larger size scale might support that SWNTs were well exfoliated with minimized amount of bundles.
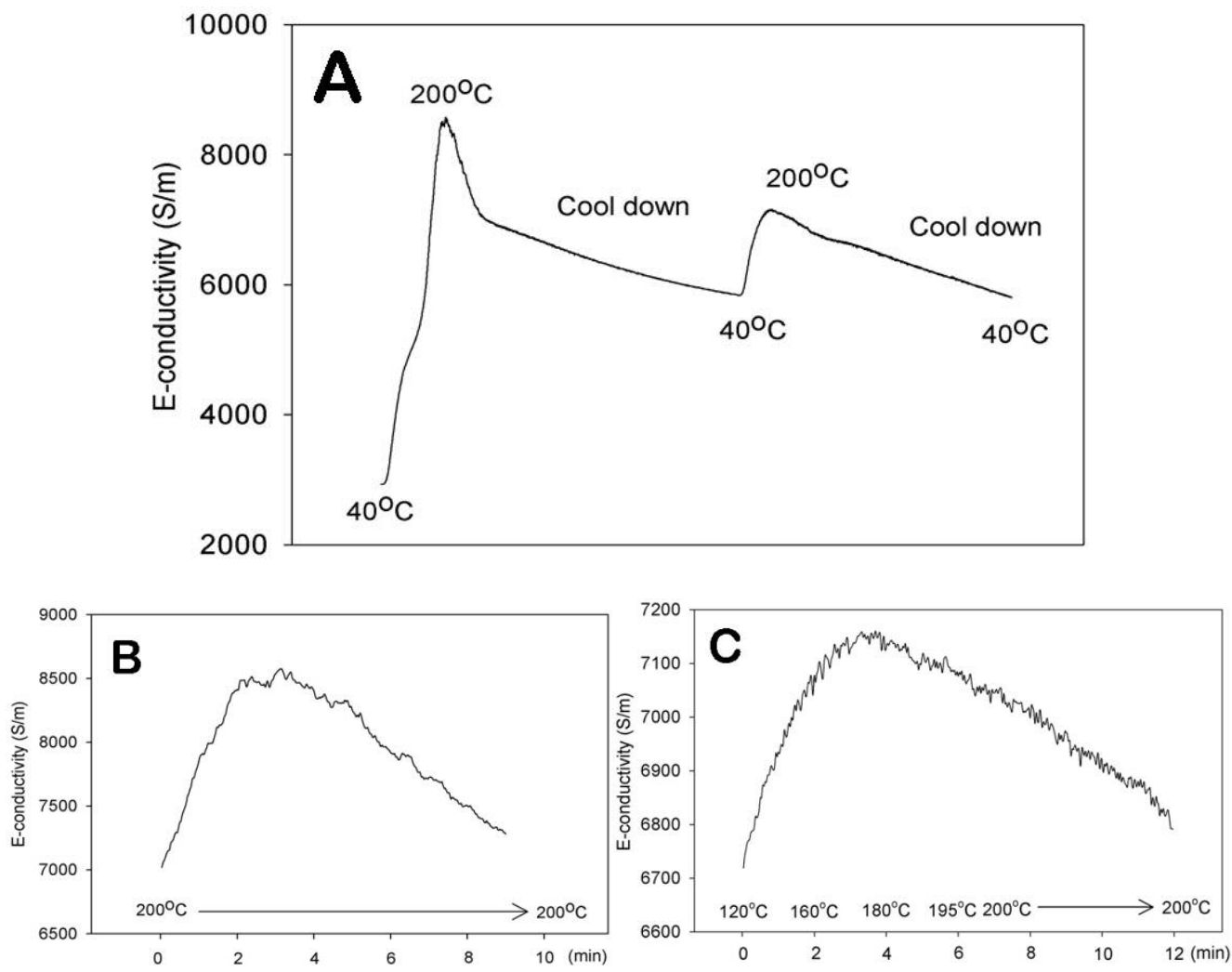

Figure S1. (A)-(C) In-situ electrical conductivity changes during heat treatment of the $[\mathrm{PVA} /(\mathrm{SWNT}+\mathrm{PSS})]_{200}$ film. (A) Electrical conductivity dramatically increases when temperature goes to $200^{\circ} \mathrm{C}$ decreasing slowly afterwards. (B) Electrical conductivity changes for the first treatment at $200^{\circ} \mathrm{C}$, the first peak in (A) as forming sticky contacts and then aggregates. (C) Electrical conductivity changes for the second treatment at $200^{\circ} \mathrm{C}$, the second peak in (A). 


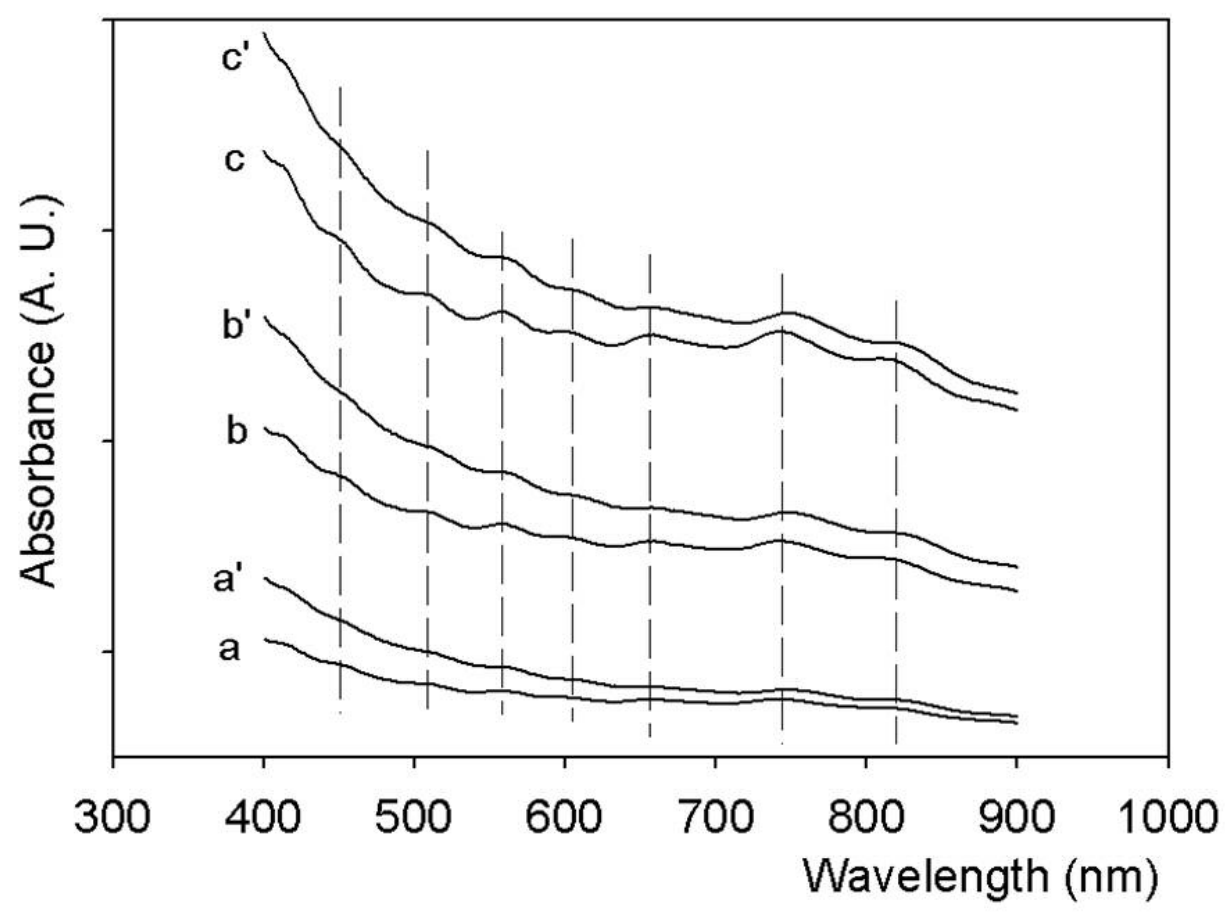

Figure S2. UV-vis spectroscopy of (a) 8 layered, (b), 12 layered, and (c) 16 layered LBL film of [PVA/(SWNT+PSS)]. (a', b', c') The same measurements of the samples were compared after $300^{\circ} \mathrm{C}$ thermal annealing treatments. Conspicuous van Hove singularity peaks in the original films were broadened and redshifted which was a good indication of permanent molecular structure rearrangement of SWNTs from extreme dispersion to tightly connected state.
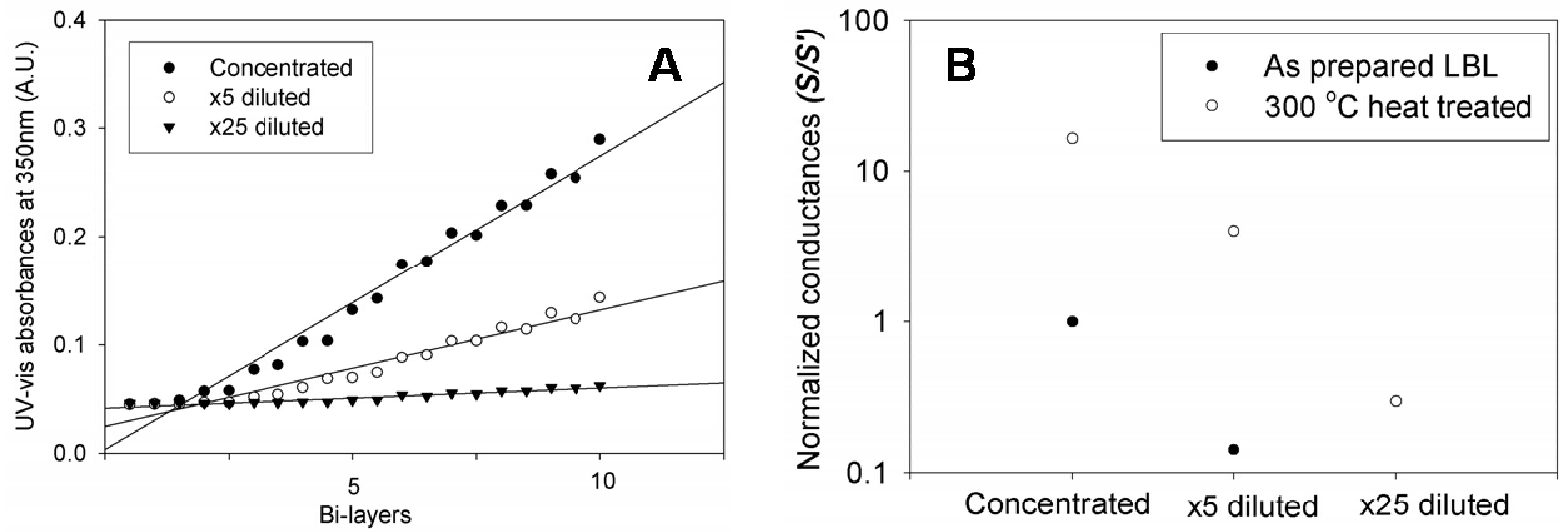

Figure S3. SWNT concentration effect during LBL assemblies (A) UV-vis spectroscopy of [PVA/diluted $(\mathrm{SWNT}+\mathrm{PSS})]_{n}$ LBL assemblies with concentrated, x5 diluted, x25 diluted SWNT solution. (B) Normalized conductance $\left(S / S^{\prime}\right)$ of the resulting 10 layered LBL film with and without heat treatment. S' indicated the conductance of as-prepared [PVA/concentrated (SWNT+PSS) $]_{10} \mathrm{LBL}$ film. 


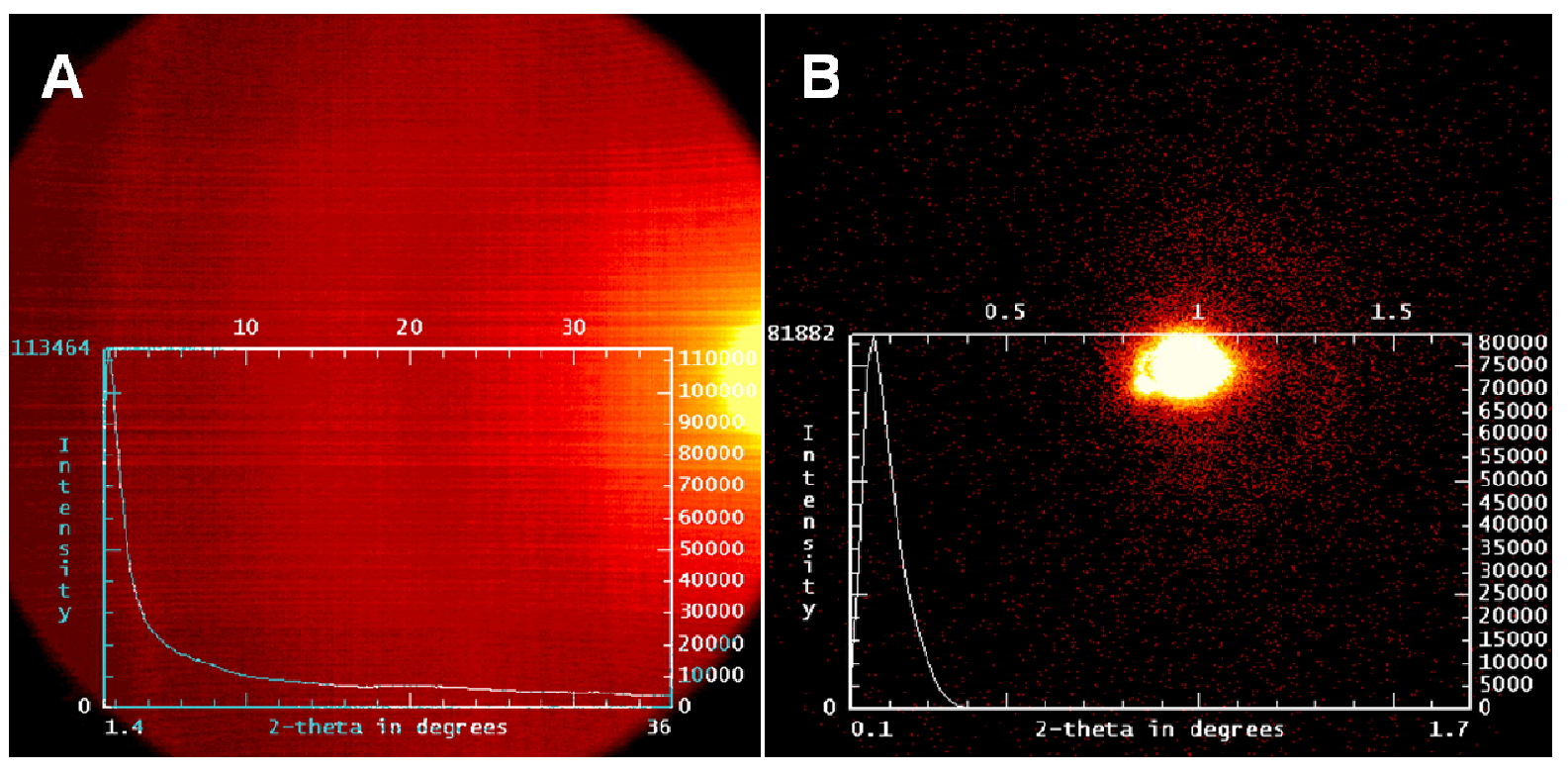

Figure S4. (A) WXRD, (B) SXRD scanning of a 200 layered SWNT LBL film. 


\section{Reference List}

1. Yamagata, Y.; Shiratori, S. Evaluation of electrical characteristics of the layer-by-layer self-assembled films after the various annealing temperatures. Thin Solid Films 2003, 438-439, 238-242.

2. Bachilo, S. M.; Strano, M. S.; Kittrell, C.; Hauge, R. H.; Smalley, R. E.; Weisman, R. B. Structure-Assigned Optical Spectra of Single-Walled Carbon Nanotubes. Science 2002, 298 (5602), 2361-2366.

3. Ramesh, S.; Ericson, L. M.; Davis, V. A.; Saini, R. K.; Kittrell, C.; Pasquali, M.; Billups, W. E.; Adams, W. W.; Hauge, R. H.; Smalley, R. E. Dissolution of Pristine Single Walled Carbon Nanotubes in Superacids by Direct Protonation. Journal of Physical Chemistry B 2004, 108 (26), 8794-8798.

4. Jacquemin, R.; Kazaoui, S.; Yu, D.; Hassanien, A.; Minami, N.; Kataura, H.; Achiba, Y. Doping mechanism in single-wall carbon nanotubes studied by optical absorption. Synthetic Metals 2000, 115 (1-3), 283-287.

5. O'Connell, M. J.; Bachilo, S. M.; Huffman, C. B.; Moore, V. C.; Strano, M. S.; Haroz, E. H.; Rialon, K. L.; Boul, P. J.; Noon, W. H.; Kittrell, C.; Ma, J.; Hauge, R. H.; Weisman, R. B.; Smalley, R. E. Band gap fluorescence from individual singlewalled carbon nanotubes. Science 2002, 297 (5581), 593-596.

6. Reich, S.; Thomsen, C.; Ordejon, P. Electronic band structure of isolated and bundled carbon nanotubes. Physical Review B: Condensed Matter and Materials Physics 2002, 65 (15), 155411-1-155411/11. 